\title{
Chronic Lithium Treatment Inhibits Pilocarpine-Induced Mossy Fiber Sprouting in Rat Hippocampus
}

\author{
David W Cadotte', Bin $\mathrm{Xu}^{2}$, Ronald J Racine ${ }^{2}$, Glenda M MacQueen', Jun Feng Wang ${ }^{3}$, Bruce McEwen ${ }^{4}$, \\ and $L$ Trevor Young*,3 \\ 'Mood Disorders Program, Department of Psychiatry and Behavioural Neurosciences, McMaster University, Hamilton, Ontario, Canada; \\ ${ }^{2}$ Department of Psychology, McMaster University, Hamilton, Ontario, Canada; ${ }^{3}$ Centre for Addiction and Mental Health, Department of \\ Psychiatry, University of Toronto, Canada; ${ }^{4}$ Laboratory of Neuroendocrinology, The Rockefeller University, New York, USA
}

\begin{abstract}
Lithium remains the gold standard in the treatment of bipolar disorder. Long-term treatment with lithium may lead to specific adaptational changes in gene expression that contribute to a neuroprotective effect. In this study, the pilocarpine model of spontaneous limbic epilepsy was used to induce mossy fiber sprouting (axonal growth of the dentate granule cells that synapse on the pyramidal cells of the CA3 region) to examine the prophylactic neuroprotective effects of lithiumin vivo. There were four groups of animals: pilocarpine treated (Pil $+/ \mathrm{Li}-$ ); pilocarpine treated followed by lithium (Pil $+/ \mathrm{Li}+$ ); lithium alone (Pil-/Li + ); control (Pil-/Li-). Timm staining was used to obtain density measurements in the stratum oriens and the inner molecular layer of the hippocampus. Mossy fiber density was higher in the pilocarpine-treated animals compared to controls. Chronic lithium following pilocarpine treatment attenuated the density of mossy fibers but lithium alone had no effect. No changes in hilar volume or neuronal number were detected using stereological procedures. The ability of lithium to attenuate activation-induced reorganization in the hippocampus provides evidence for its role as a neuroprotective agent in an in vivo model that may be relevant to its clinical effects in bipolar disorder.

Neuropsychopharmacology (2003) 28, I448-1453, advance online publication, 2 I May 2003; doi: I0.1038/sj.npp. 1300 I 89
\end{abstract}

Keywords: lithium; pilocarpine; Mossy fiber sprouting; neuroprotection; hippocampus; bipolar disorder

\section{INTRODUCTION}

The mood stabilizer, lithium, is the gold standard in the treatment of patients with bipolar disorder. Lithium must be administered chronically to treat effectively this disorder. Long-term treatment markedly improves the course of the illness, preventing relapses of either mania or depression (Poolsup et al, 2000). Although the precise mechanism of action of lithium remains unknown, this drug has a multitude of effects on monoaminergic neurotransmitters (Shiah and Yatham, 2000; Fujii et al, 2000) and signal transduction pathways (Bezchlibnyk and Young, 2002). It is widely thought that long-term changes in neuronal synaptic function are related to changes of gene expression in the brain, which appears to underlie the effects of these drugs. Chronic treatment with lithium has been found to increase the expression of proto-oncogene bcl-2 treatment in the rat

*Correspondence: Dr LT Young, Mood and Anxiety Program, Centre for Addiction and Mental Health, 250 College Street, Toronto, Ontario, Canada M5T IR8, Tel: + 416 535 850lext4749, Fax: +4I6535850।, E-mail: Trevor_Young@camh.net

Received 28 August 2002; revised 05 March 2003; accepted 07 March 2003

Online publication: 12 March 2003 at http://www.acnp.org/citations/ Npp03 | 203083/default.pdf brain and cultured cells (Chen and Chuang, 1999; Manji et al, 2000; Wei et al, 2001). Bcl-2 is an antiapoptotic factor and inhibits cytochrome $\mathrm{C}$ release from the mitochondria (Shimizu et al, 2000). This finding indicates that a neuroprotective mechanism may play an important role in the action of lithium. Recent, studies have demonstrated that chronic lithium treatment has neuroprotective effects in cellular and animal models. Dixon and Hokin (1998) reported that chronic lithium treatment upregulated mouse synaptosomal uptake of glutamate. Since accumulation of extracellular glutamate contributes to excitotoxicity, this suggests that lithium may have a protective role through the increase of glutamate uptake. Nonaka and Chuang (1998) and Nonaka et al (1998a) found that lithium protects cultured rat cerebellar, cerebral cortical, and hippocampal neurons against glutamate-induced excitotoxicity. Lithium also significantly protects cerebellar granule cells against apoptosis induced by aging of the cultures (Nonaka $e t$ al, 1998b). It was also reported that chronic treatment with lithium decreased excitotoxicity-induced striatal lesions and basal forebrain lesions in the rat (Wei et al, 2001; Pascual and Gonzalez, 1995; and Arendt et al, 1999).

A muscarinic cholinergic agonist, pilocarpine, has been shown to induce chronic spontaneous recurrent seizures and, in addition, cause mossy fiber sprouting of dentate 
granule cells beyond what is normally observed (Mello et al, 1993). Prolonged pilocarpine treatment has been shown to induce cell damage (Mello et al, 1993). Previous studies from our lab have shown that post-mortem tissue from patients diagnosed with bipolar disorder also show increased mossy fiber sprouting when compared to matched controls (Dowlatshahi et al, 2000). Since induction of mossy fiber sprouting may indicate cellular damage in the hippocampus, which may be relevant to the neuropathology of bipolar disorder, in the present study we determined if the neuroprotective effects of chronic lithium treatment extended to the reduction of pilocarpine-induced mossy fiber sprouting in the rat hippocampus.

\section{MATERIALS AND METHODS}

\section{Animals}

Male Wistar rats, obtained from Charles River NY, weighing 200-300 g were housed individually, maintained on a $12 \mathrm{~h}$ on/12 h off light cycle and provided with rat chow (Harlan Teklad) and drinking water ad libitum. Animals were acclimatized to the facilities for a period of 5 days, at which time they were randomly divided into four groups: pilocarpine-treated group $(n=8)$, pilocarpine treated with subsequent lithium treatment group $(n=8)$, lithium treatment only group $(n=8)$, and negative control group $(n=8)$. Animals receiving pilocarpine were allowed to recover for 5 days after administration. Animals were fed either a normal diet or a lithium diet for 28 days and perfused on day 29. Details are outlined below. Experimental outline is depicted in Figure 1.

\section{Pilocarpine-Induced Status Epilepticus}

Animals in the relevant groups received pilocarpine hydrochloride (Sigma) at a dose of $325 \mathrm{mg} / \mathrm{kg}$ (i.p. injection). Seizures were constantly monitored and ranked every $30 \mathrm{~s}$ according to the Racine five-stage model (Racine, 1972). Animals were required to undergo seizures at stage three or higher for $50 \mathrm{~min}$. Seizures were terminated after

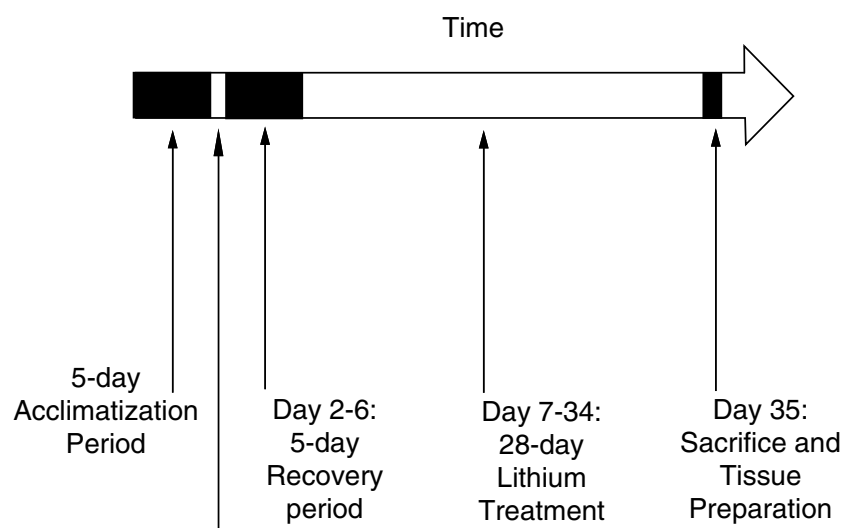

Day 1:

50 minutes of

Pilocarpine-induced

Seizure Activity.

Figure I Experimental outline.
50 min by administering Somnitol (sodium pentobarbital) at a dosage of $35 \mathrm{mg} / \mathrm{kg}$ (i.p. injection).

\section{Lithium Treatment}

Animals were provided with lithium rat chow (Harlan Teklad-lithium carbonate was added to normal rat chow at a concentration of $0.24 \%$ by weight). Chow was available to animals ad libitum, along with $0.9 \%$ saline and regular drinking water. Blood samples were taken at the time of perfusion and analyzed for lithium concentration using the Vitros Li Slide analytical method as previously reported in Wang et al (1999); briefly, lithium ions bind to a crownether dye and are detected by reflectance spectroscopy at $600 \mathrm{~nm}$.

\section{Tissue Preparation}

Animals were anesthetized with a lethal dose of sodium pentobarbital and perfused transcardially with $50 \mathrm{ml}$ of a sodium sulfide solution $\left(8.9 \mathrm{~g} \mathrm{Na}_{2} \mathrm{~S} \cdot 9 \mathrm{H}_{2} \mathrm{O}, 10.9 \mathrm{~g}\right.$ sucrose,

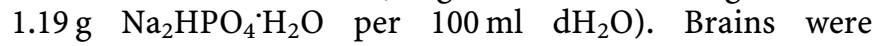
removed and immediately frozen in a solution of 2methylbutane (cooled to $-80^{\circ} \mathrm{C}$ ) and subsequently stored at $-80^{\circ} \mathrm{C}$. Six contiguous sections from Bregma $-2.30 \mathrm{~mm}$ to Bregma $-4.80 \mathrm{~mm}$ were prepared at $-20^{\circ} \mathrm{C}$. These sections were chosen because they contain hippocampus. Five equidistant slices from each of these sections were used for TIMM staining ( $40 \mu \mathrm{m}$ sections), and Cresyl Violet staining ( $60 \mu \mathrm{m}$ sections) and mounted onto gelatin-coated slides. Mounted tissue was stained according to the neoTimm method (Danscher and Zimmer, 1978) for analysis of Timm granule density or cresyl violet to determine the volume and neuronal number of the hilar region.

\section{Quantification of Timm staining}

Quantification of Timm staining was performed as previously described (Adams et al, 2002). A total of five sections $(40 \mu \mathrm{m}$ thick) were examined from each animal. Both the right and left hippocampi were analyzed as follows. Slides were examined using an objective lens $(\times 5$ magnification) by creating a digitized image with a BIOQUANT true color imaging system for Windows 98 (BioQuant-R\&M Biometrics, Inc., Tonawanda, New York) attached to a light microscope (Zeiss Axioskop, Oberkochem, Germany). A cursor (an open circle of $0.12 \mathrm{~mm}$ diameter) was placed at 16 adjacent positions along the stratum oriens of the $\mathrm{CA} 3$ region and nine adjacent positions along the inner molecular layer (IML) of the dentate gyrus; a density measurement was recorded for each cursor. An additional eight density measurements were recorded in the stratum radiatum (a region that contains few Timm granules), which provided a background measurement to account for variations across sections and slides. The relative optical density (ROD) was calculated as follows: density reading in stratum oriens (or IML)/average of background measurements recorded in the stratum radiatum. Thus, a total of 160 data points were collected in the CA3 region and 90 in the dentate gyrus region per animal. 
A four-way ANOVA $(4 \times 5 \times 2 \times 16 / 9)$ with one between variable (group) and three within variables (section (1-5 ventral to dorsal), brain hemisphere (left or right), and cursor position (1-16 in CA3, 1-9 in IML)) was conducted for the analysis of Timm granule density in the CA3 and IML regions following a method previously employed (Adams et al, 2002).

\section{Estimation of Hilar Volume and Stereological Cell Counting}

A total of 10 sections ( $60 \mu \mathrm{m}$ thick) were examined for each animal. The right and left hippocampi were analyzed as follows. Slides were examined using an objective lens $(\times 40$ magnification) by creating a digitized image with a BIOQUANT true color imaging system for Windows 98 (BioQuant-R\&M Biometrics, Inc., Tonawanda, New York) attached to a light microscope (Zeiss Axioskop, Oberkochem, Germany). The hilar area was defined by the inner edge of the granule cell layer and the lines connecting the tips of the two granule cell blades to the beginning of the pyramidal cell layer of Ammon's Horn. Hilar volume was estimated by taking the sum of the hilar area from each measured section multiplied by the distance between the sections $(420 \mu \mathrm{m})$.

Stereological cell counting (optical dissector method) was performed in accordance with previously published methods (Sterio, 1984; Abusaad et al, 1999). A brief overview, including subtle variations, is outlined below. A digital grid $(150 \mu \mathrm{m} \times 150 \mu \mathrm{m})$ was randomly positioned on the hilar area yielding from six to 29 intersections. An optical dissector was placed at each of the intersections. The counting frame of the optical dissector was a $70 \mu \mathrm{m} \times 70 \mu \mathrm{m}$ square, with a height of $10 \mu \mathrm{m}$. Cells intersecting with either the left or bottom edge of the frame were excluded while cells intersecting with either the right or top edge were included in the count. Cells that intersected the uppermost focal plane were excluded from the count. Only cells that came into focus within the $10 \mu \mathrm{m}$ height were counted. Furthermore, only cells with morphological characteristics of neurons were included in the count (distinct dendrites or irregular cell bodies with large dendrites). The estimate of the total neuronal number per hippocampus was calculated using the following equation: (neurons counted/total volume of dissectors) (estimated hilar volume).

A two-way ANOVA $(4 \times 2)$ with one between variable (group) and one within variable (brain hemisphere (left or right)) was conducted for the analysis of hilar area and cell number.

\section{RESULTS}

The onset of seizures in all animals occurred at an average of $17 \mathrm{~min}$ after the injection of pilocarpine (range: 12$24 \mathrm{~min}$ ). Animals that remained in a seizure state (status epilepticus) at stage 3 or higher for at least $50 \mathrm{~min}$ were used for further study. The average was $58 \pm 4 \mathrm{~min}$. Seizures were terminated using somnitol $(35 \mathrm{mg} / \mathrm{kg}$ i.p.) in order to prevent death of the animal or severe seizure-induced cellular damage. There was no significant change of body weight after lithium treatment for 28 days (control: $421 \pm 15 \mathrm{~g}$; pilocarpine group: $436 \pm 13 \mathrm{~g}$; lithium group: $409 \pm 8 \mathrm{~g}$; and pilocarpine plus lithium group: $438 \pm 21 \mathrm{~g}$ ). Serum lithium concentrations were $0 \mathrm{mM}(n=8), 0 \mathrm{mM}$ $(n=8), 0.92 \mathrm{mM}(n=8)$, and $1.08 \mathrm{mM}(n=6)$ in control, pilocarpine-treated, lithium-treated, and pilocarpine plus lithium-treated groups, respectively. Stereological cell counting and Timm staining were used to measure neuronal cell number and mossy fiber sprouting, respectively. As shown in Figure 2, 4 weeks after the pilocarpine treatment, no significant difference in hilar neuronal cell number or hilar volume was found among control, pilocarpine-treated (Pil), or lithium-treated (Li) animals. Additionally, there was no significant difference in the pilocarpine plus lithium-treated $(\mathrm{Pil}+\mathrm{Li})$ group (data not shown). In each case $p>0.05$. However, there was a significant increase in mossy fiber sprouting in the hippocampus 28 days after treatment with pilocarpine. Specifically, there was a significant increase in mossy fiber sprouting in the stratum oriens of the CA3 region and the IML of the dentate gyrus
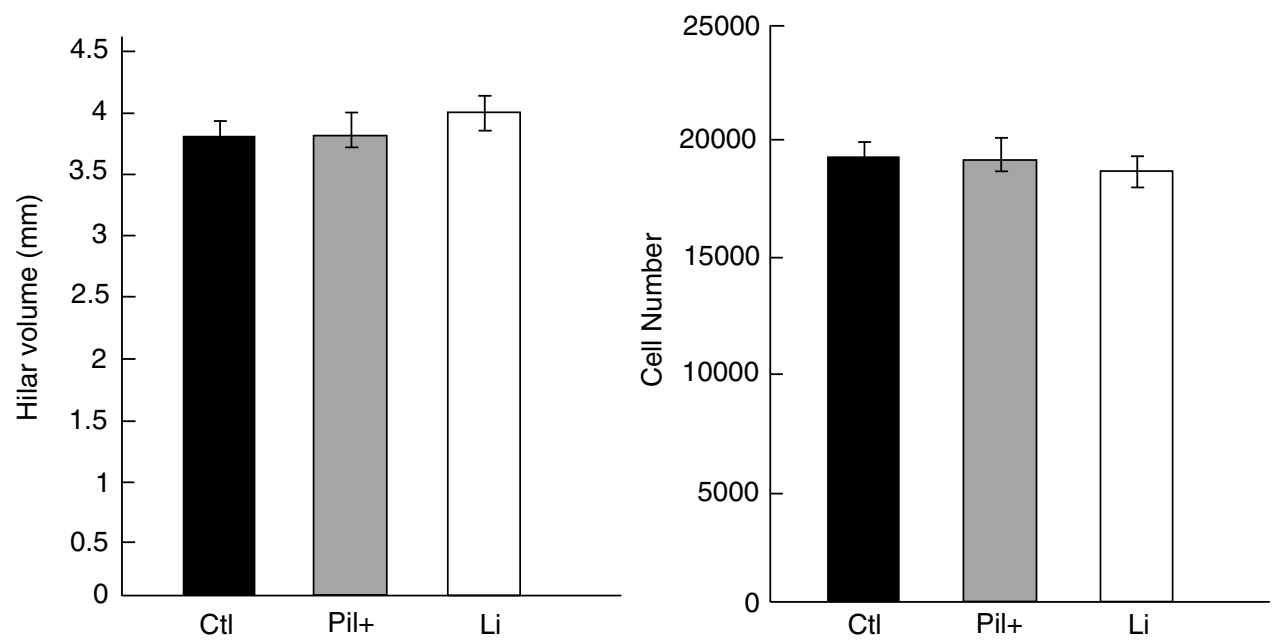

Figure 2 The effect of pilocarpine on hilar volume and cell number. Hilar volume and cell number were measured in control animals (Ctl, $n=8)$, animals subjected to pilocarpine treatment (Pil, $n=8)$, and animals treated with lithium. No difference $(p>0.05)$ was found between groups. Additionally, there was no significant difference in animals treated with both pilocarpine and lithium (data not shown). 
28 days after treatment with pilocarpine $(n=8, p<0.05)$ (Figures 3 and 4). These results suggest that treatment with pilocarpine for $50 \mathrm{~min}$ followed by somnitol resulted in a mild insult as opposed to more severe damage including cell loss in the rat brain.

To determine if the mood stabilizer lithium has any effect on sprouting induced by pilocarpine, we measured the effect of chronic lithium treatment on pilocarpine-induced mossy fiber sprouting in the stratum oriens of the CA3 region and the IML of the dentate gyrus. Although chronic treatment with lithium alone for 4 weeks had no effect on cellular outgrowth in the stratum oriens of the CA3 region and the IML of the dentate gyrus, chronic lithium treatment decreased pilocarpine-induced mossy fiber sprouting in both regions (Figures 3 and 4). To quantify the decrease of mossy fiber sprouting by lithium, we measured the density of mossy fibers. We found that chronic treatment with lithium significantly decreased ROD of mossy fibers induced by pilocarpine from $1.69 \pm 0.07$ to $1.44 \pm 0.05$ (Pil $n=8$, Pil $+\mathrm{Li} n=6 ; p<0.05$ ) in the stratum oriens of the CA3 region (Figure 3), and from $1.87 \pm 0.13$ to
$1.12 \pm 0.06$ (Pil $n=8$, Pil + Li $n=6 ; p<0.05)$ in the IML of the dentate gyrus (Figure 4 ).

\section{DISCUSSION}

In this study, we established that administration of pilocarpine under the present conditions led to induction of mossy fiber sprouting without significant cell loss in the hippocampus. It has been previously reported that treatment with pilocarpine in the range of $300-400 \mathrm{mg} / \mathrm{kg}$ can induce sustained epileptic activity such as behavioral and electrographic changes, and cause severe damage to rat brain including cell loss and mossy fiber sprouting with a high lethality rate in the first $24 \mathrm{~h}$ (Mello et al, 1993). In this study sodium pentobarbital was given 50 min after pilocarpine in order to prevent seizure-induced cell loss. We found no evidence of severe damage such as a change in hilar volume or loss of neurons as measured by Nissl staining; future studies will include TUNEL staining in an attempt to detect more subtle cellular damage that may not result in cellular death. Mossy fiber sprouting, however, was reliably
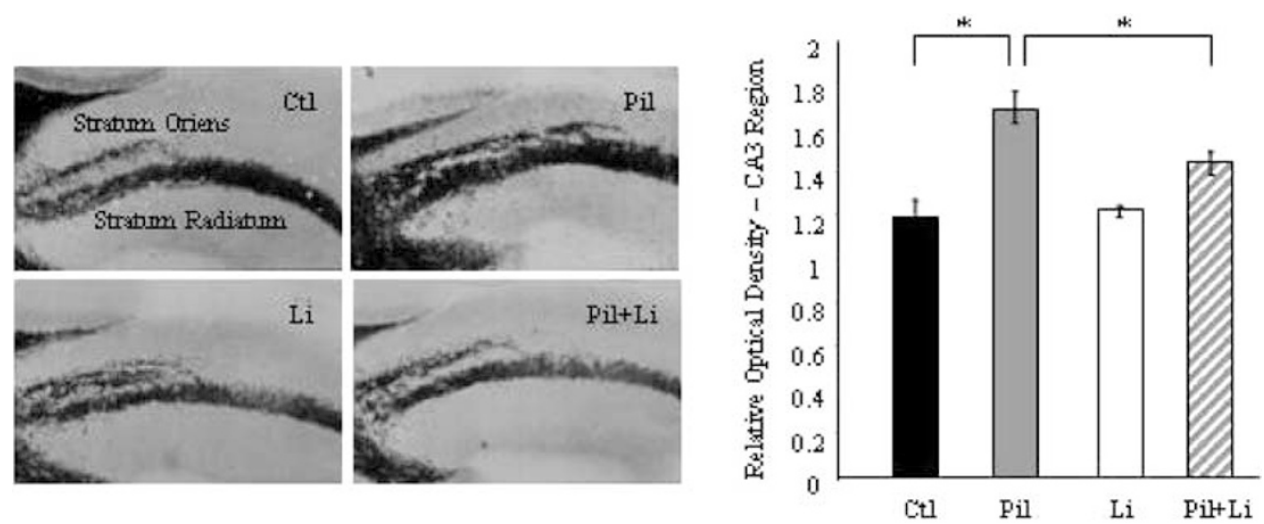

Figure 3 The effect of pilocarpine and lithium on mossy fiber density in the CA3 region. Animals treated with Pil had a significant increase in mossy fiber sprouting in the stratum oriens of the CA3 region relative to the control (CtI) group. Animals in the Pil + Li group showed a significant reduction in mossy fiber sprouting (post hoc Tukey test, $p<0.05$ ) in the stratum oriens relative to the Pil group; mossy fiber sprouting was not retuned to control levels (indicated by Ctl group). Chronic lithium treatment had no effect on mossy fiber sprouting in the absence of pilocarpine treatment; there was no significant difference between the Li group and the CtI group. Shown above are: a comparison of Timm-stained tissue from the stratum oriens region of each of the four groups and the result of statistical analysis on the density measurements.
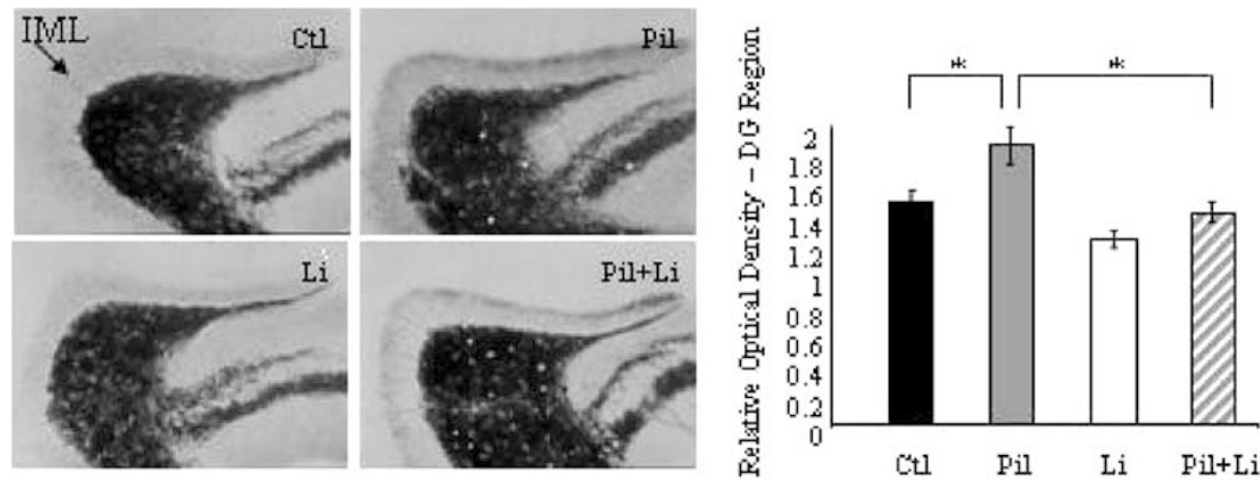

Figure 4 The effect of pilocarpine and lithium on mossy fiber density in the DG region. Pilocarpine-treated animals (Pil) had a significant increase in mossy fiber sprouting in the IML of the dentate gyrus relative to the control $(\mathrm{CtI})$ group. Animals in the Pil + Li group showed a significant reduction in mossy fiber sprouting (post hoc Tukey test, $p<0.05$ ) in the IML of the dentate gyrus relative to the Pil group, with Timm density returning to the control levels of the CtI group. Chronic lithium treatment had no effect on Timm density in the absence of pilocarpine treatment; there was no significant difference between the Li group and the Ctl group. Shown above are a comparison of Timm-stained tissue from the IML of each of the four groups and the result of statistical analysis on the density measurements. 
induced over a period of 4 weeks. Our result may suggest that this treatment only generates a mild insult without severe brain damage. Chen et al (2000) found that lithium increases the number of BrdU-positive neurons in the rat hippocampus. This finding, together with our results, suggests that lithium may have different effects on cell numbers between normal and pathological conditions. It may also suggest that in pilocarpine-treated animals, cell number represents the balance between cell death and cell regenesis.

In the present study, we determined if chronic treatment with lithium has any effect on pilocarpine-induced mossy fiber sprouting. We found that although chronic treatment with lithium at therapeutically relevant concentration alone has no effect on mossy fiber sprouting, this treatment significantly decreased mossy fiber sprouting in the hippocampus induced by pilocarpine, which suggests that chronic lithium treatment may have a neuroprotective effect against the pilocarpine insult. This result adds to the growing body of evidence suggesting that neuroprotection may be an important factor in the action of lithium as longterm treatment for bipolar disorder.

It has been well documented that mossy fibers of the dentate granule cells sprout onto, and form excitatory synapses with the basal dendrites of the CA3 pyramidal cells and the dendritic spines of the granule cells (Babb et al, 1992). A number of studies in vitro and in vivo have demonstrated that injury-induced aberrant mossy fiber sprouting is associated with granule cell hyperexcitability and spontaneous limbic seizures (Tauck and Nadler, 1985; Cronin et al, 1992; Mathern et al, 1997; Leite et al, 1996). Some researchers believe that mossy fiber sprouting renders hippocampal circuitry susceptible to the generation of prolonged seizure-like bursts of action potentials (Franck et al, 1995; Wuarin and Dudek, 1996), and that this may contribute to the onset of epileptic seizures (Babb, 1999). Excitotoxicity is a key component of kindling (Regehr et al, 1989). Kindling involves the application of a mild electrical stimulus into the limbic system. Drugs such as pilocarpine can induce similar changes in the limbic system (Cavalheiro et al, 1991).

Chronic lithium treatment attenuates mossy fiber sprouting in rat hippocampus in the pilocarpine-induced synaptic reorganization. These activation-induced effects are readily measured within the hippocampus, and occur over a period of at least 28 days. The activation-induced synaptic reorganization, mediated by pilocarpine, may be the result of an increased excitatory input to the target structures. Chronic lithium treatment may block cellular changes subsequent to excitotoxicity under in vivo conditions at therapeutic levels relevant to clinical practice. It has been reported that chronic lithium treatment increases glutamate reuptake in mouse cerebral cortical slices (Dixon and Hokin, 1998). Lithium has also been found to decrease kainic acid-increased $\left[{ }^{3} \mathrm{H}\right]$ D-aspartate uptake and glutamate decarboxylase level in the rat brain (Sparapani et al, 1997). Pretreatment with lithium prevents cell toxicity induced by glutamate and other agonists in primary neuronal cultures and also in neuroblastoma cells overexpressing GSK-3 $\beta$ (Grimes and Jope, 2001). Nonaka and Chuang reported that pretreatment with lithium could reduce the extent of ischemic damage in the cerebral cortex in adult rats
(Nonaka and Chuang, 1998). Together with our findings, this suggests that chronic treatment with lithium not only prevents, but also reverses exitotoxicity-caused brain damage. These findings are consistent with the efficacy of lithium in both the long-term prophylaxis of bipolar disorder and the treatment of acute episodes of mania and depression.

Recent post-mortem studies in subjects with bipolar disorder show increased hippocampal Timm staining compared with matched controls in anterior hippocampus (Dowlatshahi et al, 2000), suggesting that mossy fiber sprouting in the hippocampus of subjects with bipolar disorder. Since excitotoxicity of the limbic system plays an important role in mossy fiber sprouting, this finding provides evidence which is consistent with the early hypothesis of altered limbic neuronal excitability in bipolar disorder. Recent reports show that glutamate transporter, receptor and metabolite enzyme are changed in mood disorder (McCullumsmith and Meador-Woodruff, 2002). Whether such abnormalities can also be modified by treatment with lithium require further investigation

\section{ACKNOWLEDGEMENTS}

This work was supported by grants from the Theodore and Vada Stanley Foundation (LTY), the Canadian Institutes for Health Research (LTY), and the Natural Sciences and Engineering Research Council of Canada (DWC).

\section{REFERENCES}

Abusaad I, MacKay D, Zhao J, Stanford P, Collier DA, Everall IP (1999). Stereological estimation of the total number of neurons in the murine hippocampus using the optical dissector. J Comp Neurol 408: 560-566.

Adams B, Vaccarella L, Fahnestock M, Racine RJ (2002). The cholinergic system modulates kindling and kindling-induced mossy fiber sprouting. Synapse 44: 132-138.

Arendt T, Lehmann K, Seeger G, Gartner U (1999). Synergistic effects of tetrahydroaminoacridine and lithium on cholinergic function after excitotoxic basal forebrain lesions in rat. Pharmacopsychiatry 32: 242-247.

Babb TL (1999). Synaptic reorganizations in human and rat hippocampal epilepsy. Adv Neurol 79: 763-779.

Babb TL, Pretorius JK, Mello LE, Mathern GW, Levesque MF (1992). Synaptic reorganizations in epileptic human and rat kainate hippocampus may contribute to feedback and feedforward excitation. Epilepsy Res 9(Suppl): 193-202.

Bezchlibnyk Y, Young LT (2002). The neurobiology of bipolar disorder: focus on signal transduction pathways and the regulation of gene expression. Can J Psychiatry 47: 135-148.

Cavalheiro EA, Leite JP, Bortolotto ZA, Turski WA, Ikonomidou C, Turski L (1991). Long-term effects of pilocarpine in rats: structural damage of the brain triggers kindling and spontaneous recurrent seizures. Epilepsia 32: 778-782.

Chen RW, Chuang DM (1999). Long term lithium treatment suppresses p53 and Bax expression but increases Bcl-2 expression. A prominent role in neuroprotection against excitotoxicity. J Biol Chem 274: 6039-6042.

Chen G, Rajkowska G, Du F, Seraji-Bozorgzad N, Manji HK (2000). Enhancement of hippocampal neurogenesis by lithium. J Neurochem 75: 1729-1734.

Cronin J, Obenaus A, Houser CR, Dudek FE (1992). Electrophysiology of dentate granule cells after kainate-induced 
synaptic reorganization of the mossy fibers. Brain Res 573: 305310.

Danscher G, Zimmer J (1978). An improved Timm sulphide silver method for light and electron microscopic localization of heavy metals in biological tissues. Histochemistry 55: 27-40.

Dixon JF, Hokin LE (1998). Lithium acutely inhibits and chronically up-regulates and stabilizes glutamate uptake by presynaptic nerve endings in mouse cerebral cortex. Proc Natl Acad Sci USA 95: 8363-8368.

Dowlatshahi D, MacQueen G, Wang JF, Chen B, Young LT (2000). Increased hippocampal supragranular Timm staining in subjects with bipolar disorder. Neuroreport 11: 3775-3778.

Franck JE, Pokorny J, Kunkel DD, Schwartzkroin PA (1995). Physiologic and morphologic characteristics of granule cell circuitry in human epileptic hippocampus. Epilepsia 36: 543558

Fujii T, Nakai K, Nakajima Y, Kawashima K (2000). Enhancement of hippocampal cholinergic neurotransmission through 5-HT1A receptor-mediated pathways by repeated lithium treatment in rats. Can J Physiol Pharmacol 78: 392-399.

Grimes CA, Jope RS (2001). CREB DNA binding activity is inhibited by glycogen synthase kinase- 3 beta and facilitated by lithium. J Neurochem 78: 1219-1232.

Leite JP, Babb TL, Pretorius JK, Kuhlman PA, Yeoman KM, Mathern GW (1996). Neuron loss, mossy fiber sprouting, and interictal spikes after intrahippocampal kainate in developing rats. Epilepsy Res 26: 219-231.

Manji HK, Moore GJ, Chen G (2000). Lithium up-regulates the cytoprotective protein $\mathrm{Bcl}-2$ in the $\mathrm{CNS}$ in vivo: a role for neurotrophic and neuroprotective effects in manic depressive illness. J Clin Psychiatry 61(Suppl 9): 82-96.

Mathern GW, Bertram III EH, Babb TL, Pretorius JK, Kuhlman PA, Spradlin S et al (1997). In contrast to kindled seizures, the frequency of spontaneous epilepsy in the limbic status model correlates with greater aberrant fascia dentata excitatory and inhibitory axon sprouting, and increased staining for $\mathrm{N}$-methylD-aspartate, AMPA and GABA(A) receptors. Neuroscience 77: 1003-1019.

McCullumsmith RE, Meador-Woodruff JH (2002). Striatal excitatory amino acid transporter transcript expression in schizophrenia, bipolar disorder, and major depressive disorder. Neuropsychopharmacology 26: 368-375.

Mello LE, Cavalheiro EA, Tan AM, Kupfer WR, Pretorius JK, Babb TL, Finch DM (1993). Circuit mechanisms of seizures in the pilocarpine model of chronic epilepsy: cell loss and mossy fiber sprouting. Epilepsia 34: 985-995.

Nonaka S, Chuang DM (1998). Neuroprotective effects of chronic lithium on focal cerebral ischemia in rats. Neuroreport 9: 20812084 .
Nonaka S, Hough CJ, Chuang DM (1998a). Chronic lithium treatment robustly protects neurons in the central nervous system against excitotoxicity by inhibiting $N$-methyl-D-aspartate receptor-mediated calcium influx. Proc Natl Acad Sci USA 95: 2642-2647.

Nonaka S, Katsube N, Chuang DM (1998b). Lithium protects rat cerebellar granule cells against apoptosis induced by anticonvulsants, phenytoin and carbamazepine. J Pharmacol Exp Ther 286: 539-547.

Pascual T, Gonzalez JL (1995). A protective effect of lithium on rat behaviour altered by ibotenic acid lesions of the basal forebrain cholinergic system. Brain Res 695: 289-292.

Poolsup N, Li Wan PA, de Oliveira IR (2000). Systematic overview of lithium treatment in acute mania. J Clin Pharm Ther 25: 139156.

Racine RJ (1972). Modification of seizure activity by electrical stimulation. II. Motor seizure. Electroencephalogr Clin Neurophysiol 32: 281-294.

Regehr WG, Connor JA, Tank DW (1989). Optical imaging of calcium accumulation in hippocampal pyramidal cells during synaptic activation. Nature 341: 533-536.

Shiah IS, Yatham LN (2000). Serotonin in mania and in the mechanism of action of mood stabilizers: a review of clinical studies. Bipolar Disord 2: 77-92.

Shimizu S, Konishi A, Kodama T, Tsujimoto Y (2000). BH4 domain of antiapoptotic Bcl-2 family members closes voltage-dependent anion channel and inhibits apoptotic mitochondrial changes and cell death. Proc Natl Acad Sci USA 97: 3100-3105.

Sparapani M, Virgili M, Ortali F, Contestabile A (1997). Effects of chronic lithium treatment on ornithine decarboxylase induction and excitotoxic neuropathology in the rat. Brain Res 765: 164168.

Sterio DC (1984). The unbiased estimation of number and sizes of arbitrary particles using the dissector. J Microsc 134: 127-136.

Tauck DL, Nadler JV (1985). Evidence of functional mossy fiber sprouting in hippocampal formation of kainic acid-treated rats. $J$ Neurosci 5: 1016-1022.

Wang JF, Chen B, Young LT (1999). Identification of a novel lithium regulated gene in rat brain. Brain Res Mol Brain Res 70: 66-73.

Wei H, Qin ZH, Senatorov VV, Wei W, Wang Y, Qian Y, Chuang DM (2001). Lithium suppresses excitotoxicity-induced striatal lesions in a rat model of Huntington's disease. Neuroscience 106: 603-612.

Wuarin JP, Dudek FE (1996). Electrographic seizures and new recurrent excitatory circuits in the dentate gyrus of hippocampal slices from kainate-treated epileptic rats. J Neurosci 16: 44384448 . 